\title{
Estudio Comparativo de las Marcas de Dientes Producidas por Dos Pequeños Carnívoros Sudamericanos
}

\author{
Comparative Study of Tooth Marks by Two Small South American Carnivores \\ Agustina Massigoge, María A. Gutiérrez, María C. Álvarez, Cristian A. Kaufmann, \\ Daniel J. Rafuse y Mariela E. González
}

\begin{abstract}
RESUMEN
En este trabajo se presentan los primeros resultados de un estudio experimental realizado con zorro pampeano (Lycalopex gymnocercus) y gato montés (Leopardus geoffroyi) con el fin de caracterizar el patrón de marcas de dientes generado por cada carnívoro sobre restos óseos no ingeridos de un mamífero pequeño y evaluar si existen diferencias en estos patrones. Este estudio indicó que ambos carnívoros generan los mismos tipos de modificaciones y en proporciones similares; no obstante, el número promedio de marcas por espécimen es más del doble en el caso del zorro. Sobre la base de los resultados preliminares obtenidos se propone que el tamaño de los hoyuelos no parece ser un criterio diagnóstico determinante para distinguir la acción de estos dos predadores.
\end{abstract}

Palabras clave: Marcas de diente, Hoyuelos, Zorro pampeano, Gato Montés, Muestra Experimental.

\begin{abstract}
In the following paper we present the preliminary results of an experimental study performed with Pampas fox (Lycalopex gymnocercus) and Geoffroy's cat (Leopardus geoffroyi). The objectives are to characterize the tooth mark patterns generated by each carnivore on non ingested bone of a small mammal and evaluate if there exists differences in these patterns. Results indicate that both carnivores generate similar types and proportions of modifications; however, the average number of marks per specimen is double for the Pampas fox. The preliminary information obtained here indicates that the size of the pits does not appear to be a sufficient diagnostic criterion to distinguish the action of these two predators.
\end{abstract}

Key words:Tooth Marks, Pits, Pampas Fox, Geoffroy's Cat, Experimental Sample.

i INCUAPA-CONICET, Facultad de Ciencias Sociales, Universidad Nacional del Centro de la Provincia de Buenos Aires. Av. del Valle 5737, B7400JWI, Olavarría, Buenos Aires, Argentina. Correo-e: amassigo@soc.unicen.edu.ar; mgutierr@soc.unicen.edu.ar; malvarez@soc.unicen.edu.ar; ckaufman@soc.unicen.edu.ar; drafuse@soc.unicen.edu.ar; mgonzalez@soc.unicen.edu.ar

Recibido: 04-12-2012 Revisado: 05-05-2013 Aceptado: 20-12-2013 


\section{INTRODUCCIÓN}

El desarrollo de modelos actualísticos referidos a la acción de distintos agentes tafonómicos sobre conjuntos de vertebrados es fundamental para las interpretaciones arqueológicas sobre la subsistencia humana y el paleoambiente. En las últimas décadas se avanzó significativamente en el conocimiento de los patrones de modificación ósea y transporte de partes anatómicas por carnívoros. Sin embargo, es importante profundizar en el reconocimiento de la variabilidad de los patrones generados por distintas clases de carnívoros con el fin de identificar su participación en la formación de conjuntos arqueofaunísticos particulares. El tamaño de las marcas de dientes es uno de los principales criterios propuestos para diferenciar la acción de distintos tipos de carnívoros (Domínguez-Rodrigo y Piqueras 2003, Selvaggio y Wilder 200I). No obstante, si bien en los últimos años se ha producido un avance significativo en las investigaciones sobre este tema, éstas han focalizado principalmente en carnívoros africanos y europeos (e.g., Andrés et al. 2012, Domínguez-Rodrigo y Piqueras 2003, Selvaggio y Wilder 200I). En cuanto a los carnívoros sudamericanos, aunque se ha producido abundante información sobre las modificaciones que ocasionan a las carcasas y huesos (e.g., Elkin y Mondini 200I, Borrero y Martin 1996), no se ha profundizado en el estudio de la variación dimensional de las marcas de dientes considerando muestras extensas. En consecuencia, este trabajo constituye un primer acercamiento a esta problemática con el fin de generar una base de datos que contemple la variabilidad de modificaciones óseas que generan algunos de los predadores nativos que han interactuado con las sociedades prehispánicas.

En este trabajo se presentan los primeros resultados de un estudio experimental realizado con dos carnívoros sudamericanos: el zorro pampeano (Lycalopex gymnocercus) y el gato montés (Leopardus geoffroyi). Los objetivos son: I) caracterizar el patrón de marcas de dientes generado por cada tipo de carnívoro y 2) evaluar si existen diferencias en estos patrones que puedan ser reconocidas en los conjuntos fósiles. El estudio de estos dos carnívoros se enmarca en un proyecto mayor de investigaciones actualísticas orientado a resolver problemáticas tafonómicas particulares de la región pampeana (Argentina), lugar donde desarrollamos nuestras investigaciones arqueológicas.
El zorro pampeano se distribuye actualmente en el este de Bolivia, oeste de Paraguay, Uruguay, sur de Brasil y norte y centro de Argentina. Es considerado un omnívoro generalista, con una dieta dominada por mamíferos e insectos (Lucherini y Luengos Vidal 2008). La distribución del gato montés es más extensa, incluyendo Bolivia, sur de Brasil, Argentina y Chile (Ximénez 1975). Este carnívoro, como la mayoría de los félidos, se especializa en la caza de vertebrados (Manfredi et al. 2004). En la región pampeana se observa una superposición parcial en la dieta del gato montés y del zorro pampeano, siendo muy importante en ambos casos el consumo de pequeños vertebrados, principalmente roedores y aves. Además, ambos carnívoros predan sobre mamíferos más grandes (e.g., vizcacha -Lagostomus maximus-, liebre europea -Lepus europaeus-, armadillos -Dasipodidae-; Lucherini y Luengos Vidal 2008, Manfredi et al. 2004). Aunque aún no hay estudios conclusivos, la ocurrencia de carroñeo parece ser más alta en el caso del zorro pampeano que en el del gato montés (Novaro et al. 2000). En cuanto a su tamaño, el zorro pampeano (machos 5,9 kg; hembras 4,7 kg; Lucherini y Luengos Vidal 2008) presenta un promedio de peso corporal ligeramente superior al gato montés (machos 4,8 kg; hembras 3,6 kg; Lucherini et al. 2006).

Algunas de las presas de estos carnívoros formaron parte de la dieta de las poblaciones humanas prehispánicas del Holoceno de la región pampeana y de regiones aledañas (Álvarez et al. 2013, Escosteguy et al. 20I2, Quintana y Mazzanti 20II, entre otros). $\mathrm{Si}$ se considera que el carroñeo es una estrategia alimentaria más común en la dieta de los cánidos con respecto a los félidos, sería esperable que el impacto sobre el registro arqueofaunístico sea mayor en el caso de los zorros. Estos carnívoros podrían modificar, destruir y desplazar restos óseos descartados por los humanos, así como incorporar al depósito huesos de sus presas a través de restos no ingeridos y fecas; mientras que en el caso de los gatos, si bien se considera que pueden ocurrir los mismos procesos, el mayor impacto estará referido a la incorporación de restos de animales que forman parte de su dieta.

\section{MATERIALESY MÉTODOS}

Las muestras analizadas provienen de un estudio experimental realizado en el Zoológico Municipal La Máxima de la ciudad de Olavarría (provincia 
de Buenos Aires, Argentina). Este estudio consistió en alimentar con conejos (Oryctolagus cuniculus) a dos gatos monteses y a un zorro. A cada clase de carnívoro se le ofreció un total de 10 presas completas en distintos eventos. Las presas pesaban unos $3 \mathrm{~kg}$ en promedio, correspondiendo a las categorías subadulto y adulto. En cada evento de alimentación se colocó una presa completa (segmentada en mitad anterior y posterior por protocolo del zoológico), en el caso del gato, y media, en el caso del zorro, dentro del hábitat de cada carnívoro. Luego de tres días, se recolectaron los restos óseos no ingeridos y las fecas; durante este lapso, no se brindó ningún otro alimento a los carnívoros. La elección de la presa se basó en que su tamaño es similar al de los mamíferos pequeños explotados por las poblaciones prehispánicas de la región.

Los resultados presentados en este trabajo corresponden a los restos no ingeridos, los cuales se analizaron considerando las siguientes variables: I) tipo de marca, 2) su frecuencia en las distintas porciones de los elementos y 3) tamaño de los hoyuelos. Cada espécimen fue examinado a ojo desnudo y bajo una lupa estereoscópcia de 20-40x.

Las marcas fueron clasificadas en hoyuelos, perforaciones, arrastres, bordes crenulados y furrows (Binford 198I, Haynes 1983). Asimismo, se tomaron fotografías de los hoyuelos utilizando un microscopio digital Dino-Lite (AnMo Electronics Corporation). Las imágenes digitales fueron empleadas para medir las marcas, utilizando el programa Image] (Rasband I 997 20I2) según la metodología propuesta por DelaneyRivera y coautores (2009). Se calculó el área, el largo máximo y el ancho máximo a partir del perímetro dibujado de cada marca. Es importante aclarar que un porcentaje considerable de hoyuelos presentes en las muestras aún no han sido medidos. Esto se debe, por un lado, a que no todos los especímenes con marcas han sido fotografiados $y$, por otro lado, a que ca. $43 \%$ de la muestra analizada -sobre todo aquellas marcas muy pequeñas y superficiales- no pudo ser medida debido a dificultades técnicas en la obtención de las imágenes'. La selección de los hoyuelos entre los tipos de marcas se debe a que sus dimensiones se ajustan más estrechamente a las del diente del predador $y$, en consecuencia, se correlacionan de modo más fuerte con el tamaño del carnívoro involucrado (Domínguez-Rodrigo y Piqueras 2003, Selvaggio y Wilder 200I).

\section{RESULTADOS}

La muestra de restos óseos no ingeridos recuperada en el hábitat de los gatos asciende a 792 especímenes, de los cuales alrededor del 55\% pudieron ser identificados anatómicamente (Tabla I). En el caso del zorro, la muestra recuperada es de 639 especímenes, de los cuales aproximadamente un $86 \%$ fueron identificados a nivel anatómico. $\mathrm{Si}$ bien en este trabajo no discutiremos la destrucción ósea y la representación de partes esqueletarias resultante, es pertinente mencionar que ambos predadores ocasionan una importante pérdida de huesos, particularmente de costillas y vértebras, impactando significativamente en el Número Mínimo de Individuos inicial de conejos (Rafuse et al. 20l4).

\begin{tabular}{|l|c|c|}
\hline & Gato montés & Zorro pampeano \\
\hline Especímenes (n) & 792 & 639 \\
\hline Especímenes identificados anatómicamente (n) & 438 & 547 \\
\hline Especímenes con marcas* (n) & 81 & 72 \\
\hline Total de marcas (n) & 511 & 1048 \\
\hline Hoyuelos (n; \%) & $278(54,4)$ & $611(58,3)$ \\
\hline Perforaciones (n; \%) & $76(14,9)$ & $64(6,1)$ \\
\hline Arrastres (n; \%) & $125(24,5)$ & $368(35,1)$ \\
\hline Bordes crenulados (n; \%) & $30(5,9)$ & $4(0,4)$ \\
\hline Furrows (n; \%) & $2(0,4)$ & $1(0,1)$ \\
\hline Promedio marcas/espécimen** & 6,3 & 14,5 \\
\hline
\end{tabular}

Tabla 1: Características generales de las muestras. ${ }^{*}$ Sólo se consideran los identificados a nivel anatómico, ${ }^{* \star}$ Sólo se consideraron los especímenes con marcas

Table 1: General characteristics of the samples, ${ }^{*}$ Including only the anatomical identified specimens, ${ }^{* *}$ Including only the specimens with tooth marks 
Considerando los especímenes identificados anatómicamente, se observa que un $18,5 \%$ exhibe al menos algún tipo de marca en la muestra del gato y un $13,2 \%$ en la del zorro (Tabla I). Se registra una importante diferencia en el número de marcas producidas por cada carnívoro. El total de marcas identificadas en la muestra del zorro asciende a 1048 y el promedio de marcas por espécimen es de 14,5. Por su parte, el total de marcas registradas en la muestra del gato montés es de $5 \mathrm{II}$ y el promedio de marcas por espécimen es de 6,3 (Tabla I).

En cuanto a la distribución anatómica, se observa una ausencia de marcas sobre carpianos, astrágalos y tarsianos menores en ambos casos. Por otra parte, algunos elementos fueron afectados en baja frecuencia, tales como metapodios (gato: ca. $6 \%$; zorro: ca. $5 \%$ ), falanges (gato: ca. $5 \%$; zorro: $0 \%$ ) y calcáneos (gato: ca. I4\%; zorro: 0\%). Los elementos más modificados son: pelvis (gato: ca. $76 \%$; zorro: $100 \%$ ), escápula (100\% en ambas muestras), huesos largos (gato: ca. 62\%; zorro: ca. $83 \%$ ) y mandíbulas (gato: ca. 57\%; zorro: 70\%) (Figura I).

En ambos casos, el tipo de modificación ósea más abundante son los hoyuelos, seguido por los arrastres. Las perforaciones y los bordes crenulados se presentan en bajo porcentaje, siendo más comunes en la muestra del gato montés. Los furrows están prácticamente ausentes en ambas muestras (Tabla I; Figura 2).

En la Tabla 2 se presentan los resultados de las distintas variables relevadas en la muestra de hoyuelos. Hasta el momento se midió un total de 84 hoyuelos producidos por gato montés (ca. $30 \%$ de la muestra de hoyuelos producidos por este carnívoro) y 289 por zorro pampeano (ca. $47 \%$ de la muestra). Siguiendo a otros autores que consideran que el tamaño de las marcas está influido, en parte, por la densidad ósea y el grosor relativo del hueso (Domínguez-Rodrigo y Piqueras 2003, Selvaggio y Wilder 200I); se estratificó la muestra en dos grupos: elementos axiales y cinturas por un lado (incluyendo mandíbula, pelvis, escápula y vértebras) y huesos largos por otro. Estos últimos fueron a su vez subdivididos en porciones menores de acuerdo al espesor del tejido cortical y a la distribución del tejido trabecular (i.e., tejido esponjoso, tejido cortical delgado ${ }^{2}$, tejido cortical; Selvaggio y Wilder 200I).
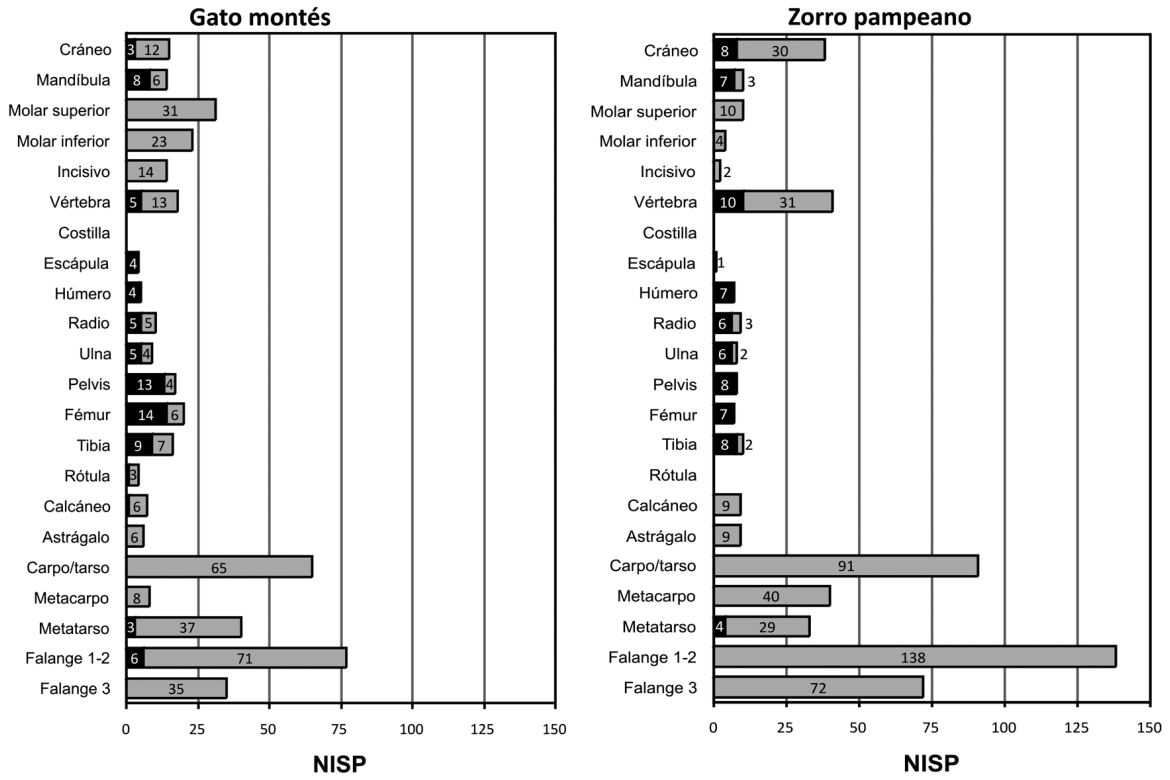

Figura 1: Distribución anatómica de modificaciones óseas. En gris se presenta el total de especímenes; en negro se presenta el total de especímenes con marcas. Los números sobre las barras representan las frecuencias absolutas.

Figure 1: Anatomical distribution of bone modifications. In grey is the total number of specimens; in black is the total number of specimens with marks. Numbers on the bars show the absolute frequencies. 

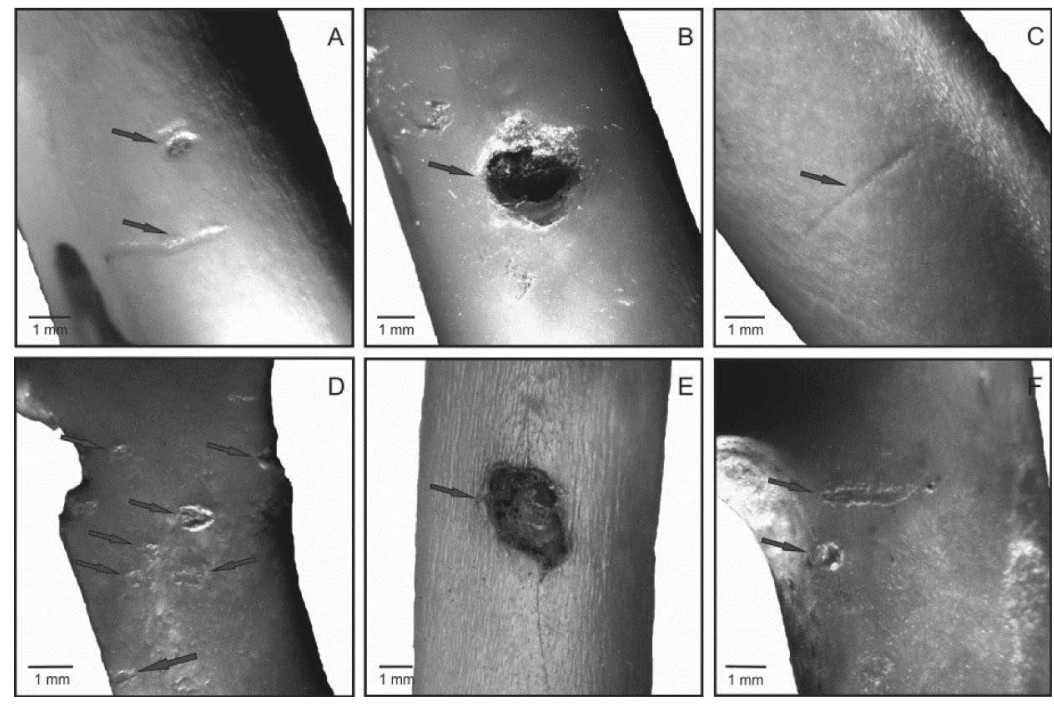

Figura 2: Detalle de algunas modificaciones generadas por gato montés (arriba) y zorro pampeano (abajo). A: Hoyuelo y arrastre sobre diáfisis de fémur; B: Perforación sobre diáfisis de tibia; C: Arrastre sobre diáfisis de fémur; D: Hoyuelos sobre diáfisis de fémur; E: Perforación sobre diáfisis de tibia; F: Arrastre y hoyuelo sobre pelvis.

Figure 2: Detail of some modifications generated by Geoffroy's cat (upper) and Pampas fox (lower). A: Femur shaft with pit and score; B: Tibia shaft with puncture; C: Femur shaft with score; D: Femur shaft with pits; E: Tibia shaft with puncture; F: Pelvis with score and pit.

\begin{tabular}{|c|c|c|c|c|c|c|c|}
\hline & $\mathbf{n}$ & Media & IC 95\% (LI) & IC 95\% (LS) & DS & Min & Max \\
\hline Gato montés_TCD_largo & 30 & $\mathrm{I}, \mathrm{I}$ & 0,953 & 1,246 & 0,393 & 0,492 & 2,099 \\
\hline Gato montés_TCD_ancho & 30 & 0,783 & 0,672 & 0,88 & 0,287 & 0,374 & $\mathrm{I}, 446$ \\
\hline Gato montés_TCD_área & 30 & 0,749 & 0,558 & 0,939 & 0,511 & 0,145 & 2,384 \\
\hline Zorro pampeano_TCD_largo & 103 & 0,862 & 0,796 & 0,929 & $0,34 \mathrm{I}$ & 0,276 & $|, 94|$ \\
\hline Zorro pampeano_TCD_ancho & 103 & 0,557 & 0,515 & 0,6 & 0,217 & 0,226 & $\mathrm{I}, 325$ \\
\hline Zorro pampeano_TCD_área & 103 & 0,421 & 0,355 & 0,486 & 0,334 & 0,049 & 1,946 \\
\hline Gato montés_TC_largo & 28 & 0,943 & 0,797 & 1,088 & 0,375 & 0,375 & 2,056 \\
\hline Gato montés_TC_ancho & 28 & 0,621 & 0,536 & 0,707 & 0,22 & 0,327 & 1,159 \\
\hline Gato montés_TC_área & 28 & 0,503 & 0,368 & 0,638 & 0,348 & 0,1 & 1,639 \\
\hline Zorro pampeano_TC_largo & 83 & 0,909 & 0,823 & 0,995 & 0,394 & 0,328 & 2,199 \\
\hline Zorro pampeano_TC_ancho & 83 & 0,587 & 0,537 & 0,636 & 0,227 & 0,304 & 1,879 \\
\hline Zorro pampeano_TC_área & 83 & 0,469 & 0,373 & 0,565 & 0,44 & 0,082 & 3,245 \\
\hline Gato montés_AX_largo & 25 & 0,994 & 0,847 & 1,142 & 0,357 & 0,472 & $\mathrm{I}, 833$ \\
\hline Gato montés_AX_ancho & 25 & 0,655 & 0,577 & 0,732 & 0,187 & 0,358 & 1,004 \\
\hline Gato montés_AX_área & 25 & 0,547 & $0,44 I$ & 0,683 & 0,329 & 0,133 & I,405 \\
\hline Zorro pampeano_AX_largo & 101 & 0,943 & 0,858 & 1,029 & 0,434 & 0,286 & 2,734 \\
\hline Zorro pampeano_AX_ancho & 101 & 0,6 & 0,553 & 0,646 & 0,234 & 0,227 & I,872 \\
\hline Zorro pampeano_AX_área & 101 & 0,51 & 0,41 & 0,611 & 0,509 & 0,051 & 3,822 \\
\hline
\end{tabular}

Tabla 2: Datos estadísticos para el largo máximo, ancho máximo y área (en $\mathrm{mm}$ ) de los hoyuelos estratificados por porción ósea. TCD: tejido cortical delgado; TC: tejido cortical; IC: intervalo de confianza; LI: límite inferior, LS: límite superior; DS: desvío estándar; Min: valor mínimo; Max: valor máximo

Table 2: Descriptive statistics for the length, width and area (in $\mathrm{mm}$ ) of the pits on different bone elements and portions. TCD: thinning cortical bone; TC: cortical bone; IC: confidence interval; LI: lower boundary; LS: upper boundary; DS: standard deviation; Min: minimum value; Max: maximum value 
Con respecto a los hoyuelos medidos sobre huesos largos, de acuerdo a las tres dimensiones consideradas, aquellos producidos por gato montés son ligeramente mayores, tanto sobre tejido cortical como sobre tejido cortical delgado ${ }^{3}$ (Tabla 2; Figura $3 \mathrm{~A}$ ). Sin embargo, estas diferencias son estadísticamente significativas sólo en esta última porción, donde no se observa superposición entre ambas muestras en los intervalos de confianza del 95\% para ninguna de las medidas consideradas (Tabla 3). A pesar de estas diferencias estadísticas, si se compara el rango total de datos, se observa una importante superposición en las dimensiones de los hoyuelos producidos por ambos carnívoros, lo cual indica que algunas marcas producidas por zorro pueden ser mayores a otras producidas por gato montés (Figura 3A). En cuanto a los hoyuelos sobre elementos axiales y cinturas, no se observan diferencias significativas entre las marcas generadas por gato montés y zorro pampeano en ninguna de las dimensiones consideradas (Tablas 2 y 3; Figura $3 B)$.

\begin{tabular}{|l|l|c|}
\hline Largo & Porción anatómica & $\boldsymbol{p}$ \\
máximo & Tejido cortical & 0,4886 \\
& Tejido cortical delgado & 0,002093 \\
& Elem. axiales y cinturas & 0,2984 \\
Ancho & Tejido cortical & 0,3829 \\
& Tejido cortical delgado & $9,29 \mathrm{E}-05$ \\
& Elem. axiales y cinturas & 0,1202 \\
& Tejido cortical & 0,396 \\
& Tejido cortical delgado & 0,0002436 \\
& Elem. axiales y cinturas & 0,1412 \\
\hline
\end{tabular}

Tabla 3: Resultados de prueba U de Mann-Whitney comparando las dimensiones de los hoyuelos producidos por gato montés y zorro pampeano sobre distintas porciones anatómicas

Table 3: Mann-Withney $U$ test results comparing pit size on different elements and portions for Geoffroy's cat and Pampas fox
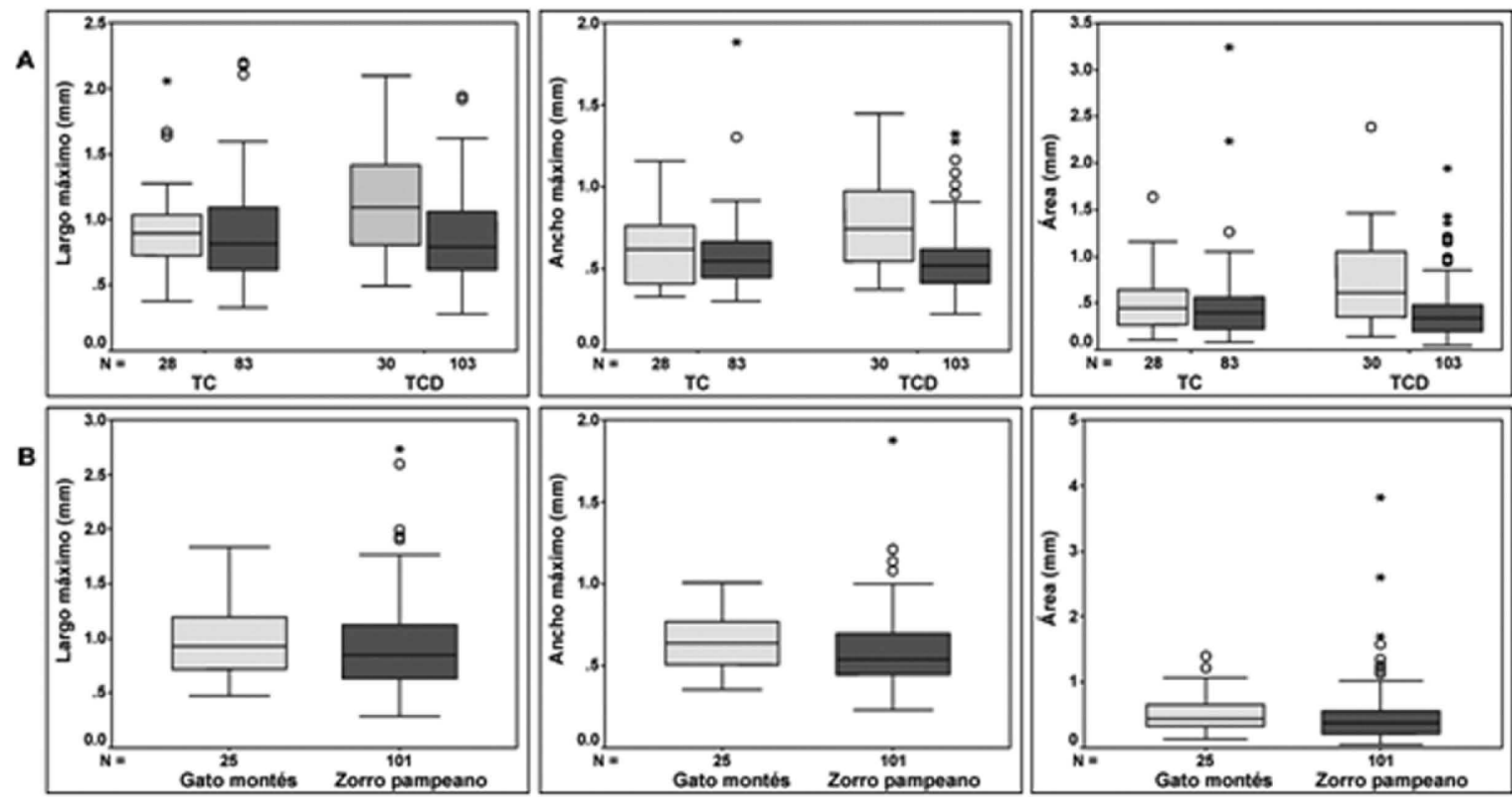

Figura 3: Box plots que muestra el rango de variación en el largo máximo, ancho máximo y área de los hoyuelos producidos por gato montés (gris claro) y zorro pampeano (gris oscuro). A: hoyuelos sobre distintas porciones de huesos largos (TC: tejido cortical; TCD: tejido cortical delgado); B: hoyuelos sobre elementos axiales y cinturas. $N$ es el número de casos disponible para cada submuestra.

Figure 3: Box plots showing the range of variation in maximum length, maximum width, and maximum area of the pits produced by Geoffroy's cat (shown in light grey) and Pampas fox (shown in dark grey). A: pits located on distinct portions of long bones (TC: cortical bone; TCD: thinning cortical); B: pits located on axial elements and girdles. $N$ is the number of instances for each subsample. 


\section{DISCUSIÓNY CONCLUSIONES}

Los datos obtenidos hasta el momento permiten realizar una primera aproximación a la caracterización de los patrones de modificaciones óseas producidos sobre mamíferos pequeños por zorro pampeano y gato montés. La evaluación de las marcas a partir de las variables consideradas permite delinear algunas tendencias:

a) Ambos carnívoros generan los mismos tipos de modificaciones y en proporciones similares, dominando en ambos casos los hoyuelos y arrastres.

b) El porcentaje de especímenes con marcas de dientes es mayor en el caso del gato montés aunque el número promedio de marcas por espécimen es mayor en el caso del zorro. La primera característica podría estar vinculada a que en la muestra no ingerida del zorro existe una mayor frecuencia de elementos del autopodio, los cuales tienden a ser menos modificados (Figura I). De este modo, si se excluyen del cálculo dichos huesos, el porcentaje de especímenes con marcas en el caso del zorro es superior al del gato $(43,9 \%$ y $35,5 \%$, respectivamente). Con respecto al promedio de marcas por espécimen, el resultado obtenido era el esperable teniendo en cuenta el comportamiento de alimentación de ambos predadores. Estudios sobre carnívoros de mayor tamaño indican que, en general, los félidos mastican los huesos en menor grado que los cánidos (Van Valkenburgh 1996).

c) El patrón de distribución anatómica de las marcas es similar entre ambos predadores, siendo la escápula, la pelvis, la mandíbula y los huesos largos los elementos más afectados.

d) El tamaño de las marcas no es un criterio diagnóstico determinante para distinguir la acción de estos dos predadores. Sólo las dimensiones de los hoyuelos sobre huesos largos -particularmente en el tejido cortical delgado- exhiben diferencias significativas entre gato montés y zorro pampeano. A pesar de estas diferencias, se observa una importante superposición en los rangos de tamaño de los hoyuelos producidos por los dos carnívoros, indicando que no es posible utilizar este atributo para asignar una marca aislada a uno u otro. Nuestros resultados concuerdan con estudios previos realizados por otros colegas, que han mostrado la dificultad de diferenciar taxa particulares por el tamaño de las marcas de dientes (Andrés et al. 2012, Delaney-Rivera et al. 2009, Domínguez-Rodrigo y Piqueras 2003).

Este estudio ha mostrado que los tipos de marcas, distribución y dimensiones de las mismas en las muestras tomadas en condiciones particulares (e.g., cautividad, presa muerta) no son criterios útiles para discriminar la actividad de zorro pampeano y gato montés. No obstante, como fue planteado previamente por otros investigadores (Domínguez-Rodrigo y Piqueras 2003, Selvaggio y Wilder 200 I), se considera que el tamaño de los hoyuelos sobre huesos largos puede ser potencialmente válido para reconocer la acción de una clase de tamaño de carnívoro, información que también resulta de utilidad para evaluar los efectos que pudo tener dicho predador en la formación de un conjunto faunístico. Un criterio que demostró ser más efectivo para diferenciar la acción de los dos carnívoros es la frecuencia de marcas por espécimen, la cual fue significativamente más alta en el caso del zorro. Aun así, creemos que es necesario complementar estos datos con el análisis de muestras naturalistas donde se elimine la posibilidad de sesgos en la alimentación resultantes de la situación de encierro del predador.

Agradecimientos: Este estudio es desarrollado dentro del programa de investigaciones de INCUAPA-CONICET (Facultad de Ciencias Sociales, UNCPBA) y es financiado por CONICET (PIP II2-20080I-0029I) y ANPCyT (PICT 088I4). Agradecemos especialmente al personal del Parque Zoológico La Máxima, particularmente a Horacio Grandt y Agustín Venzi. Agradecemos a los evaluadores anónimos por sus atinadas sugerencias. Las ideas vertidas en este trabajo son de exclusiva responsabilidad de los autores.

\section{NOTA}

La muestra de huesos de conejo no ingeridos por zorro pampeano con hoyuelos asciende a 53 especímenes (considerando sólo huesos largos y elementos axiales), de los cuales fueron fotografiados 37 . De estos últimos, sólo pudieron medirse 289 hoyuelos de un total de 493 , por limitaciones técnicas. Con respecto a la muestra de restos óseos de conejos no ingeridos por gato montés, los especímenes con hoyuelos son 42 , de los cuales fueron fotografiados 22. De estos últimos, sólo pudieron medirse 84 hoyuelos de un total de 159. 
2 Siguiendo a Selvaggio y Wilder (200I), se considera tejido cortical delgado a la porción de diáfisis más cercana a la epífisis donde el tejido cortical es más débil.

3 Debido a que la muestra de marcas sobre tejido esponjoso es muy pequeña ( $I$ hoyuelo para gato montés y 2 para zorro pampeano), esta porción no se incluyó en el presente análisis.

\section{BIBLIOGRAFÍA}

Álvarez, M. C., A. P. Alcaraz, M. A. Gutiérrez y G. A. Martínez. 2013. "Análisis zooarqueológico del sitio Paso Otero 4 (partido de Necochea). Aportes a la discusión de los modelos de subsistencia de la región pampeana". Intersecciones en Antropología 14:383-398.

Andrés, M., A. O. Gidna, J. Yravedra y M. Domínguez-Rodrigo. 2012. "A study of dimensional differences of tooth marks (pits and scores) on bones modified by small and large carnivores". Archaeological and Anthropological Sciences 4 (3): 209-219.

Binford, L.R. 198I. Bones: Ancient Men and Modern Myths. Academic Press, Nueva York.

Borrero, L. A. y F. M. Martin. 1996. "Tafonomía de carnívoros: un enfoque regional". En Arqueología. Sólo Patagonia, editado por J. Gómez Otero, pp. 189-198. CENPAT-CONICET, Puerto Madryn.

Delaney-Rivera, C., T.W. Plummer, J.A. Hodgson, F. Forrest, F. Hertel y J. S. Oliver. 2009."Pits and pitfalls: taxonomic variability and patterning in tooth mark dimensions". Journal of Archaeological Science 36: 2597-2608.

Domínguez-Rodrigo, M. y A. Piqueras. 2003. "The use of tooth pits to identify carnivore taxa in tooth-marked archaeofaunas and their relevance to reconstruct hominid processing behaviours". Journal of Archaeological Science 30: I385|39|.

Elkin, D. y M. Mondini. 200I. "Human and Small Carnivore Gnawing Damage on Bones - an Exploratory Study and its Archaeological Implications". En Ethnoarchaeology of Andean South America. Contributions to Archaeological Method and Theory, editado por L.A. Kuznar, pp. 255-265. International Monographs in Prehistory, Ann Harbor.
Escosteguy, P. D., M. Salemme y M. I. González. 2012. "Myocastor coypus ("coipo", Rodentia, Mammalia) como recurso en los humedales de la Pampa boanerense: patrones de explotación". Revista del Museo de Antropología 5: 13-30.

Haynes, G. 1983. “A guide for differentiating mammalian carnivore taxa responsible for gnaw damage to herbivore limb bones". Paleobiology 9: 164-172.

Lucherini, M. y E. M. Luengos Vidal. 2008.“'Lycalopex gymnocercus (Carnivora: Canidae)”. Mammalian Species 820: I-20.

Lucherini, M., C. Manfredi, E. Luengos, F. Dias Mazim, L. Soler y E. B. Cananave. 2006. "Body mass variation in the Geoffroy's cat (Oncifelis geoffroyi)". Revista Chilena de Historia Natural 79: I69-I74.

Manfredi, C., M. Lucherini, A. D. Canepuccia y E. B. Casanave. 2004. "Geographical variation in the diet of Geoffroy's cat (Oncifelis geoffroyi) in pampas grassland of Argentina". Journal of Mammalogy 86 (6): I I I I-I I I5.

Novaro, A. J., M. C. Funes y R. S. Walker. 2000. "Ecological extinction of native preys of a carnivore assemblage in Argentine Patagonia". Biological Conservation 92: 25-53.

Quintana, C. y D. Mazzanti. 2011. "Las Vizcachas Pampeanas (Lagostomus Maximus, Rodentia) en la Subsistencia Indígena del Holoceno Tardío de las Sierras de Tandilia Oriental (Argentina)". Latin American Antiquity 22: 253-270.

Rafuse, D. J., M. E. González, C. A. Kaufmann, M. C. Álvarez, M. A. Gutiérrez y A. Massigoge. 2014. "Análisis comparativo de los patrones de modificaciones óseas de dos carnívoros sudamericanos: el gato montés (Leopardus geoffroyi) y el zorro pampeano (Lycalopex gymnocercus).Aportes para la identificación de la acción de pequeños carnívoros en el registro arqueológico". Magallania 42 (I): | 65- 184.

Rasband, W. S. 1997-2012. Image,, U. S. National Institutes of Health, Bethesda, Maryland, Estados Unidos. http:// imagej.nih.gov/ij/.

Selvaggio, M. M. y J. Wilder. 200I. "Identifying the Involvement of Multiple Carnivore Taxa with Archaeological Bone Assemblages". Journal of Archaeological Science 28: 465-470.

Van Valkenburgh, B. 1996. "Feeding behavior in freeranging, large African carnivores”. Journal of Mammalogy 77 (I): 240-254.

Ximénez, A. 1975. "Felis geoffroyi”. Mammalian Species 54: $1-4$. 